\title{
Effectiveness of Two Methods for Preparation of Autologous Platelet-Rich Plasma: An Experimental Study in Rabbits
}

\author{
Maria J. H. Nagata ${ }^{a}$ \\ Michel R. Messora ${ }^{b}$ \\ Flávia A. C. Furlaneto ${ }^{a}$ \\ Stephen E. Fucinia \\ Alvaro F. Bosco ${ }^{a}$ \\ Valdir G. Garcia ${ }^{a}$ \\ Tatiana M. Deliberador ${ }^{c}$ \\ Luiz G. N. de Melod
}

\section{ABSTRACT}

Objectives: The purpose of this study was to compare the quantity and quality of platelets in platelet-rich plasma (PRP) samples prepared using either the single- or the double-centrifugation protocol.

Methods: Ten adult white New Zealand rabbits were used. Ten $\mathrm{ml}$ of blood were drawn from each animal via cardiac puncture. Each blood sample was divided into two equal parts for PRP preparation: $5 \mathrm{ml}$ of blood were centrifuged according to a single-centrifugation protocol (Group I), and $5 \mathrm{ml}$ were centrifuged according to a double-centrifugation protocol (Group II). Manual platelet counts were performed on the whole blood and PRP samples of each group. Smears were also done on all samples in order to see the morphology of the platelets. The data obtained in the manual platelet count were submitted to statistical analysis (repeated measures ANOVA, Tukey, $P<.05$ ).

Results: The average whole blood platelet count was $446,389 / \mu \mathrm{l}$. The PRP samples in Group II presented an average platelet amount significantly higher than that of Group I $11,986,875 \pm 685,020 / \mu \mathrm{l}$ and $781,875 \pm 217,693 / \mu \mathrm{l}$, respectively). The PRP smears from Group II were the only one to present platelets with altered morphology (75\% of the smears). A few lymphocytes with increased cytoplasm were observed in the PRP smears of both Groups I (25\% of the smears) and II (62.5\% of the smears).

Conclusions: Within the limits of this study, it can be concluded that the double-centrifugation protocol resulted in higher platelet concentrations than did the single-centrifugation protocol. However, the double-centrifugation protocol caused alterations in platelet morphology and was more sensitive to small processing errors. (Eur J Dent 2010;4:395-402)

Key words: Platelet count; Platelet-rich plasma; Centrifugation; Blood.

\footnotetext{
Professor, DDS, PhD, Division of Periodontics, Department of Surgery and Integrated Clinic,

Dental School of Araçatuba, São Paulo State University, UNESP, Brazil.

b Professor, DDS, PhD, Division of Integrated Clinic, Dental School of Lavras, Lavras University Center, UNILAVRAS, Brazil.

Professor, DDS, PhD, Program in Clinical Dentistry, Positivo University, Brazil.
}

\section{d DDS, PhD, Private Practice, Goiânia, Brazil.}

- Corresponding author:

Michel Reis Messora

Rua Barão do Rio Branco,

342 - apt 204

CEP: 37200-000 Lavras, MG, Brazil

Phone: +553538213132

E-mail: michel_messoradaterra.com.br 


\section{INTRODUCTION}

Platelet-rich plasma (PRP) is a platelet concentrate in a small plasma volume. PRP also contains fibrin, fibronectin and vitronectin. These proteins are capable of enhancing cell adhesion and act like a matrix for the formation of bone, connective tissue and epithelium. ${ }^{1}$ Platelets contain growth factors in their alfa granules, such as platelet-derived growth factor, transforming growth factor- $\beta$ and insulin-like growth factor-I which, once released, may positively regulate the wound-healing process. ${ }^{1}$

The therapeutic administration of PRP ranges from the treatment of multiple musculoskeletal disorders to the regeneration and accelerated healing of a wide range of tissues. However, there are controversies in the literature regarding the potential benefits of this procedure. Although some authors have reported significant improvements in tissue healing and bone formation using platelet-rich plasma, ${ }^{2-6}$ others have not. ${ }^{7-10}$ Such discrepancies are probably related to the lack of suitable standardization and definition of the different PRP preparations. ${ }^{11}$ The protocols and surgical techniques used in the preparation and administration of the PRP differ widely. ${ }^{12,13}$ Variations in some key properties of the PRP, such as the platelet concentration, can greatly influence the different biological effects. ${ }^{11}$

According to Whitman et $\mathrm{al}^{14}$ and Marx et al, ${ }^{15}$ PRP is prepared by the discontinuous cell separation method from a blood volume of approximately $450 \mathrm{ml}$. However, this protocol for PRP preparation involves sophisticated technology and a large blood volume, limiting its use to blood transfusion medical centers or hospital facilities. ${ }^{16}$ Therefore, equipment capable of producing small amounts of PRP has become available commercially. This seems to have resulted in higher acceptance by patients because of lower cost and the feasibility of being conducted in non-hospital environments. ${ }^{17}$

Several simplified protocols for the preparation of PRP have been developed to facilitate its clinical application. 2,18,19 However, these protocols must be followed carefully, taking several technical issues into account. Basically, the centrifugation process for PRP preparation must be sterile and precisely suited to platelet separation from red blood cells and their sequestration in high concentrations without any damage or lysis that might trigger the premature release of GFs. ${ }^{1}$ It must be remembered that the PRP platelet count is strictly dependent on the type of protocol used for PRP preparation. ${ }^{20}$ According to Marx, ${ }^{1}$ PRP should have a platelet concentration 300 to $400 \%$ greater than that of the whole blood in order to be considered a "therapeutic PRP." Lower concentrations are reportedly unreliable in enhancing wound healing, while higher concentrations have not been shown to further enhance wound healing. ${ }^{21}$

Marx et al ${ }^{15}$ stated that a double-centrifugation technique is necessary to truly concentrate platelets from autologous blood. On the other hand, Anitua $^{2,22}$ described using a single-spin technique, although the platelet concentrations obtained by this procedure were not reported. In spite of the amount of platelets, Anitua has demonstrated enhancement and acceleration of bone regeneration and more rapid and predictable soft tissue healing in future sites for implants that were treated with PRP prepared according to a single-spin technique. ${ }^{2}$ Other authors have reported obtaining platelet concentrations of $356 \%$ using the singlespin technique. ${ }^{23}$

The purpose of this study was to compare the quantity and quality of platelets in PRP samples prepared using either the single- or the doublecentrifugation protocol in an animal model.

\section{MATERIALS AND METHODS}

\section{Experimental model}

The experimental protocol was approved by the São Paulo State University, UNESP, Dental School of Araçatuba Institutional Animal Care and Use Committee. Ten adult male white New Zealand rabbits weighing 2.8 to $4 \mathrm{~kg}$ (UNESP, Dental School of Araçatuba, Animal Care Unit) were used in this study. An endoparasitosis control program, including vaccination, vermifungal treatment regimen and balanced diet was instituted. The animals were housed in individual cages at room temperature and were fed solid chow and water ad libitum.

\section{Anesthesia}

The animals were anesthetized by intramuscular injection of xylazine $(10 \mathrm{mg} / \mathrm{kg}$ body weight) and ketamine $(1.5 \mathrm{mg} / \mathrm{kg}$ body weight). 


\section{PRP preparation}

a) Cardiac puncture and blood sample collection: A $10 \mathrm{ml}$ volume of autologous blood was drawn from each animal via cardiac puncture. Each blood sample was divided into two $5 \mathrm{ml}$ vacuum tubes containing $10 \%$ sodium citrate. One milliliter of the blood was separated for platelet count. The two $5 \mathrm{ml}$ blood samples were randomly assigned to one of the following groups: Group I, in which the PRP was prepared according to a single-centrifugation protocol, ${ }^{2}$ or Group II, in which the PRP was prepared according to a double-centrifugation protocol. ${ }^{19}$

b) Protocol for PRP preparation in Group I: The separation of the blood cell elements was performed using a laboratory centrifuge (Beckman J-6M Induction Drive Centrifuge, Beckman Instruments Inc., Palo Alto, CA, USA). The blood samples were centrifuged at $160 \mathrm{G}$ for 6 minutes at room temperature resulting in three basic components: red blood cells (bottom of the tube), PRP (middle of the tube) and platelet-poor plasma (PPP) (top of the tube). One milliliter of PPP was pipetted and discarded. Next, a mark was made $2 \mathrm{~mm}$ below the line separating the middle component from the lower component of the tube. All content above this point (approximately $1.2 \mathrm{ml}$ ) was pipetted and comprises the volume of PRP.

c) Protocol for PRP preparation in Group II:

First centrifugation: The separation of the blood cell elements was performed using a laboratory centrifuge (Beckman J-6M Induction Drive Centrifuge, Beckman Instruments Inc., Palo Alto, CA, USA). The tubes were centrifuged at $160 \mathrm{G}$ for 20 minutes at room temperature resulting in two basic components: blood cell component (BCC) in the lower fraction and serum component (SEC) in the upper fraction.

Second centrifugation: A mark was made 6 $\mathrm{mm}$ below the line that separated the BCC from the SEC. To increase the total amount of platelets collected for the second centrifugation, all content above this point was pipetted and transferred to another $5 \mathrm{ml}$ vacuum tube without anticoagulant. The sample was then centrifuged again at $400 \mathrm{G}$ for 15 minutes resulting in two components: SEC and PRP. The PRP (approximately $0.5 \mathrm{ml}$ ) was separated from the SEC.

\section{Platelet count study}

The platelets in the whole blood and PRP samples from Groups I and II were counted manually in the Neubauer chamber. Brecher liquid was used to lyse the erythrocytes. Two parameters, based in part on the study by Tamimi et al, ${ }^{21}$ were evaluated for the PRP samples: platelet increase compared to whole blood and platelet concentration. These values were calculated using the following equations:

$\%$ platelet increase over whole blood $=$ Platelet count of PRP - Platelet count of whole blood

Platelet count of whole blood

Platelet concentration $(\%)=$

Platelet count of PRP

Platelet count of whole blood

PRP and whole blood were also used to perform smears which were stained with "Panótico Rápido LB" (LaborClin, Pinhais, PR, Brazil) in order to reveal the morphology of the blood cells and platelets. The platelet counts and the analysis of the platelet morphology were performed by a veterinary hematologist blinded to the PRP preparation protocol used.

\section{Statistical analysis}

The significance of differences between the whole blood and the PRP platelet counts as well as between the PRP platelet counts of Groups I and II was determined by an analysis of variance (repeated measures ANOVA), followed by a post hoc Tukey's test when the ANOVA suggested a significant difference between groups $(P<.05)$.

Pearson's correlation coefficient $\left(r_{p}\right)$ was used to demonstrate the relationship between the platelet counts from the PRP and whole blood samples of Groups I and II.

\section{RESULTS}

Platelet count study

Two animals were excluded from the study after blood centrifugation for PRP preparation in Group I. The low PPP volume in these samples did not allow for preparation of the PRP as specified in the single-centrifugation protocol.

The PRP smears of both Groups I and II showed higher concentrations of platelets than their re- 
spective whole blood smears (Figure 1). In both groups, numerous packed red blood cells were seen surrounding compact round platelet aggregates. Only the PRP smears from Group II presented platelets with altered morphology. From eight PRP smears analyzed from each group, six PRP smears of Group II showed some deformed platelets suggestive of elongated pseudopodia (Figure 2). A few lymphocytes with increased cytoplasm were observed in the PRP smears of both Groups I (2 PRP smears) and II (5 PRP smears) (Figure 3).

The average whole blood platelet count was $454,68 \pm 181,538 / \mu \mathrm{l}$. The average PRP platelet count in Group I was $781,875 \pm 217,693 / \mu \mathrm{l}$, whereas it was $1,986,875 \pm 685,020 / \mu$ l in Group II (Figure 4). Significant differences were observed between the platelet counts from the PRP samples of Groups I and II $(P<.05)$. Significant differences between the whole blood and PRP platelet counts were observed only in Group II ( $P<.05)$. A significant correlation was observed between the platelet count from the whole blood and PRP samples in Groups I $\left(r_{p}=0.93, P=0.002\right)$ and II $\left(r_{p}=0.89, P=0.0010\right)$

Platelet concentration of PRP and platelet increase over whole blood samples between Groups I and II are depicted in Table 1. The percentage increase in the platelet count of PRP compared to whole blood was higher in Group II than in Group I (Table 1).

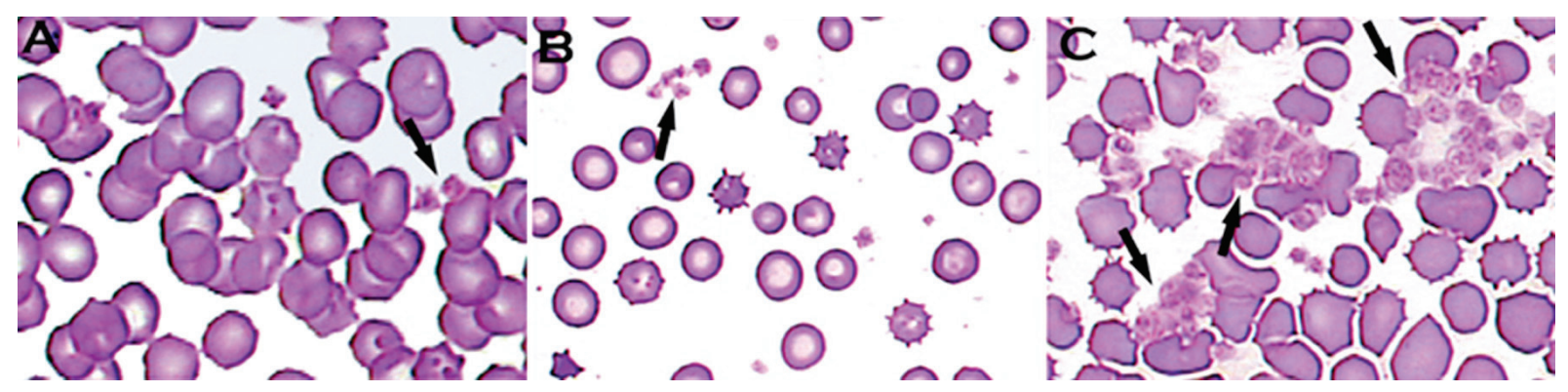

Figure 1. Whole blood and PRP smears showing platelet aggregates (arrows). Whole blood (A), PRP smear of Group I (B) and PRP smear of Group II (C). (Panótico Rápido LB staining; original magnification $\times 1000$ )
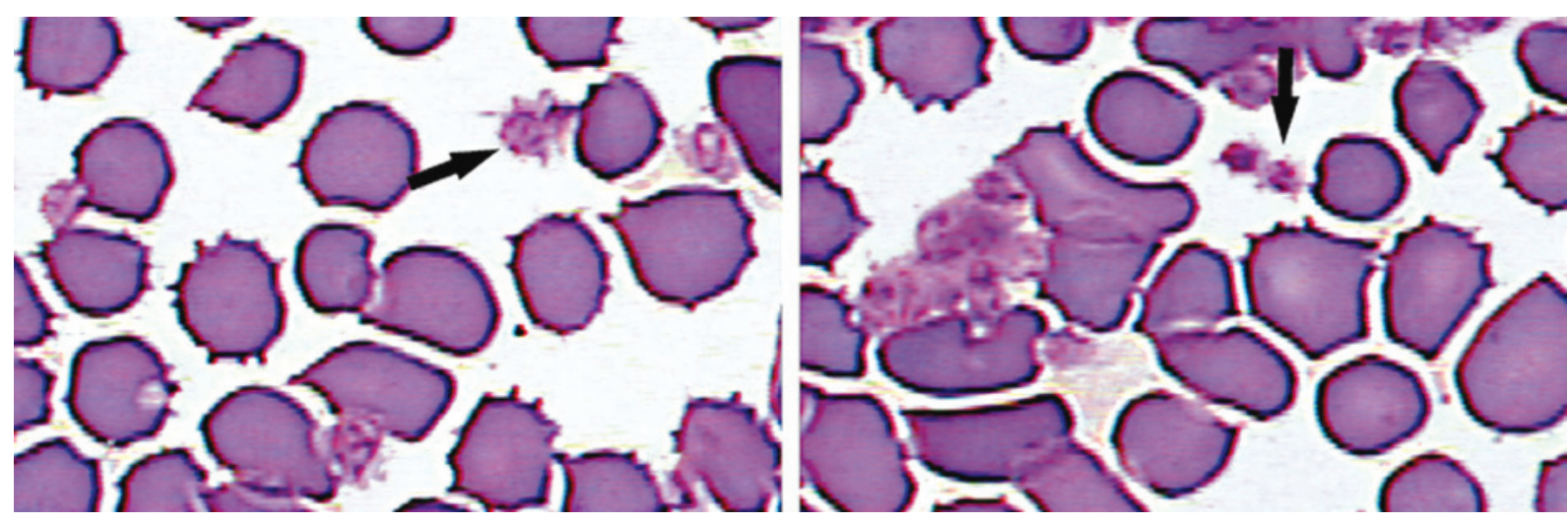

Figure 2. PRP smears (Group II) showing platelets (arrows) with altered morphology. (Panótico Rápido LB staining; original magnification x1000)

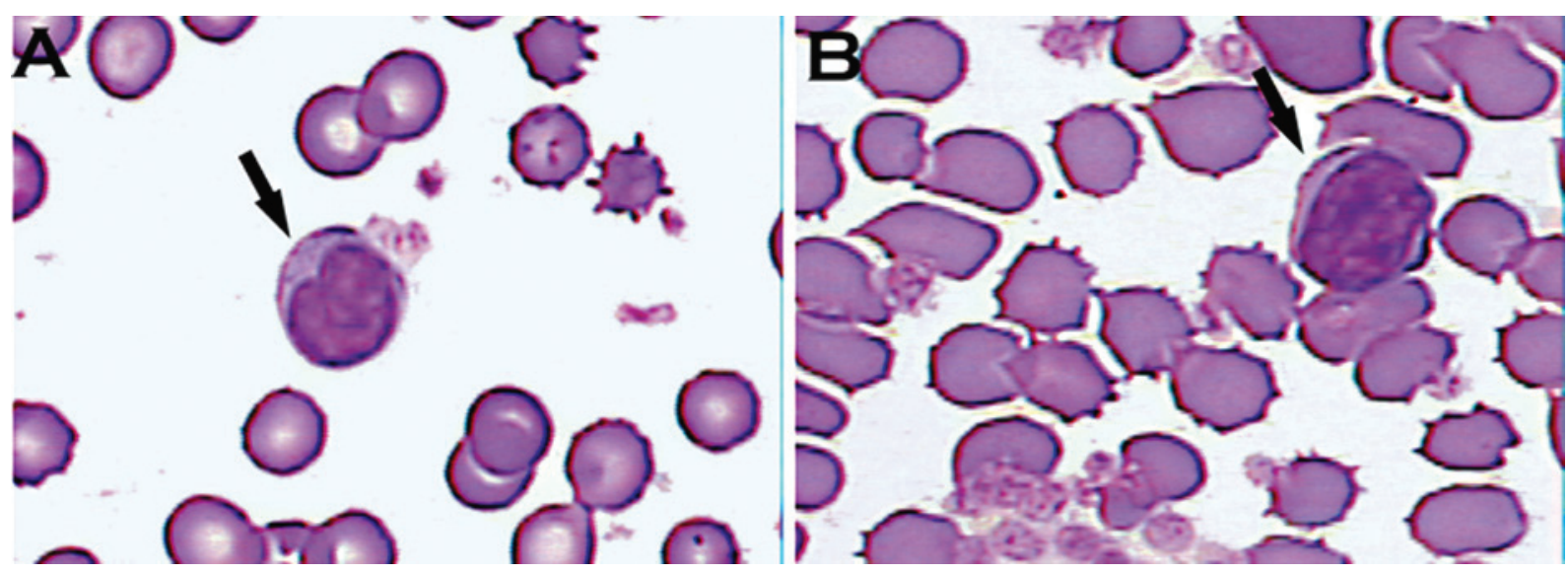

Figure 3. PRP smears of Group I (A) and Group II (B) showing white blood cells (arrows). (Panótico Rápido LB staining; original magnification x1000) 


\section{DISCUSSION}

Different PRP preparation protocols may result in varying platelet concentrations, and thus different biologic effects may occur. ${ }^{21,24,25}$ The platelet count should be one of the key factors by which to standardize studies investigating the regenerative capacity of PRP. ${ }^{26}$ In addition, qualitative alterations in the platelets may also affect the regenerative potential of PRP. ${ }^{27}$ According to Marx, ${ }^{1}$ platelets damaged or rendered nonviable by the protocol used to process the PRP will not secrete bioactive growth factors. Thus, the resulting outcome may be disappointing. The present study evaluated both the quantity and quality of platelets in PRP samples prepared according to two different protocols.

According to Marx, a "therapeutic PRP" should present approximately one million platelets per microliter in humans, considering that the whole blood contains approximately 200,000 \pm 75,000 platelets per microliter. Therefore, a "therapeutic PRP" is one that has an average percentage increase of approximately $400 \%$ in the platelet count. Studies in dogs and rabbits have demonstrated that a 4-fold increase in the platelet concentration was effective in accelerating bone healing. ${ }^{25,28}$ In the present study, the animals showed an average whole blood platelet count of 446,389 platelets per microliter, which is within the normal range for the animal model used. ${ }^{29}$ In this study, only the double centrifugation protocol used in Group II produced a "therapeutic PRP" (Table 1).

The low platelet concentrations obtained in the PRP samples of Group I are most likely attributable to the fact that only a single centrifugation was used in this protocol. According to Marx, clinicians should use either a double-centrifugation technique or some other FDA approved system specifically developed for PRP preparation. ${ }^{1,30}$

In the double-centrifugation protocol, the first spin (called the hard spin) separates the red blood cells from the plasma, which contains the platelets, the white blood cells and the clotting factors. The second spin (called the soft spin) further separates the platelets, white blood cells and few remaining red blood cells from the plasma. In contrast, a single spin would not produce a true PRP. Instead, it would produce a mixture of PRP and PPP, resulting in disappointingly low platelet concentrations. ${ }^{1}$ In the present study, the double-

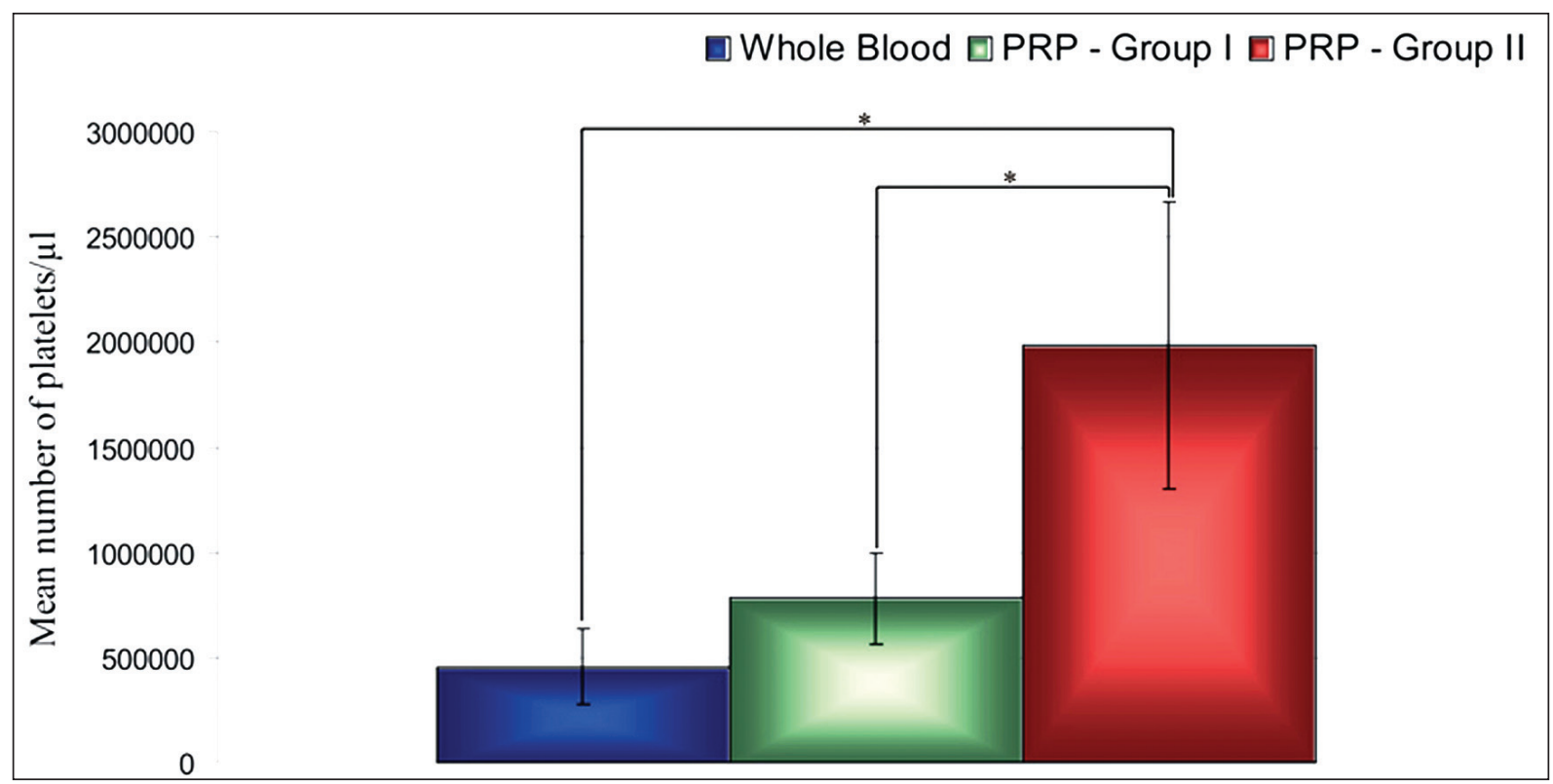

Figure 4. Whole blood and PRP platelet counts and Standard Deviations for Groups I and II, as well as the results of the comparison among the groups. * indicates statistically significant difference $(P<.05)$.

Table 1. Platelet concentration of PRP and platelet increase over whole blood samples between groups I and II.

\begin{tabular}{lcc} 
& Group I & Group II \\
\hline Parameter & Mean \pm SD & Mean \pm SD \\
\hline Platelet concentration of PRP (platelets/ $\mu$ l) & $180.73 \pm 31.01$ & $447.68 \pm 58.00$ \\
Platelet increase over the whole blood $(\%)$ & $80.73 \pm 31.01$ & $347.68 \pm 58.00$ \\
\hline
\end{tabular}


centrifugation protocol yielded increases in platelet concentrations similar to those obtained in humans with two systems developed specifically for PRP preparation (PCCS, 3i, Inc., Palm Beach Gardens, FL, USA and "SmartPrep," Harvest Technologies, Plymouth, MA, USA). ${ }^{1}$

In addition to the number of centrifugations, there are several important factors that should be considered with regard to the PRP preparation method chosen. The force of gravity $(G)$ used in the centrifugation process is one. An increase in $\mathrm{G}$ may result in higher platelet concentrations. ${ }^{3}$ In the present study, blood centrifugation at $400 \mathrm{G}$ (Group II) provided a significantly higher platelet concentration than did blood centrifugation at 160 G (Group I). However, it is important to remember that increasing the $G$ used for the centrifugation may prematurely activate the platelets. ${ }^{31}$ Platelets can also be prematurely activated by excessive pipetting ${ }^{32,33}$ or by the type of anticoagulant used during the preparation of PRP. ${ }^{29,30}$

The effects of the protocol to prepare PRP on the quality of the concentrated platelets were evaluated in this study through platelet smear analysis. Smears are valid to evaluate many parameters that are indicative of platelet function, such as changes in morphology, size, shape, staining characteristics, degree of activation and clump formation, distribution of granules and appearance of vacuoles. ${ }^{34}$ In the present study, the PRP smears of Group II showed some deformed, round-shaped platelets which may indicate premature platelet activation. Premature activation would lead to an early release of growth factors, causing them to move to the top of the tube when centrifuged. Thus, the resultant PRP would be poor in growth factors. ${ }^{21}$ Since the levels of growth factors present in the PRP samples of the present study were not measured, further studies are required to confirm this hypothesis.

Additionally, the PRP smears of both groups had some lymphocytes with increased cytoplasm. This observation is consistent with activation of the immune response resulting in increased synthesis and release of cytokines by white blood cells, which could prematurely activate platelet degranulation and thus cause a decrease in the number of growth factors present at the time the PRP is placed. ${ }^{21}$ A recent in vivo study lends support to this hypothesis. Radiographic and histomorpho- metric analyses were conducted to evaluate bone healing in the calvaria of rabbits following the use of autogenous grafts combined with PRP obtained by either a single- or double-centrifugation protocol. ${ }^{35}$ The combination of PRP with autogenous bone did not improve the initial healing process, irrespective of the PRP preparation protocol used. According to the authors, both protocols may have harmed platelets or caused them to degranulate prematurely, thus reducing the effect of PRP.

From a clinical standpoint, a second important consideration is that the platelet concentration process used to prepare the PRP be very reliable. ${ }^{20}$ Both protocols evaluated in the present study showed a high correlation between the whole blood and PRP platelet counts (Group I $r_{p}=0.93$ and Group II $-r_{p}=0.89$ ). Therefore, the level of correlation between these two parameters suggests that the whole blood platelet count can be used as an estimate of the platelet count likely to be produced by the two protocols evaluated in this study.

Significant variations in hematocrit between subjects must also be considered when choosing a method of PRP preparation. The single-centrifugation protocol used in this study did not result in any PRP being produced in two animals whose samples had a total plasma volume smaller than $1 \mathrm{ml}$ after blood centrifugation because the protocol requires that $1 \mathrm{ml}$ of PPP be discarded. After pipetting and discarding the upper layer of PPP, there was only the RBC layer left. However, the double-centrifugation protocol was able to produce PRP in both animals.

It is important to emphasize that, in this study, the final volume of the PRP samples in Groups I and II were different $11.2 \mathrm{ml}$ and $0.5 \mathrm{ml}$, respectivelyl. According to Efeoglu et al, ${ }^{29}$ the platelet concentration may be higher in a small volume of plasma than in a greater volume of plasma. The findings of the present study corroborate this statement because the protocol used in Group II led to a significantly greater amount of platelets in PRP samples than the one obtained in PRP samples of Group I.

The selection of the animal model used is also very important because some have blood volumes too small to produce autologous PRP, thus necessitating the use of donor blood for the PRP preparation protocol. The use of homologous PRP could 
compromise the reliability of the results of those studies because, according to Marx, ${ }^{1}$ only autologous PRP is true PRP. In the present study, PRP was prepared using a small blood volume $(10 \mathrm{ml})$. The total amount of blood that can be collected from an adult New Zealand rabbit without risking its life is limited to $15 \mathrm{ml} .{ }^{29}$ Therefore, the amount of blood collected in this study allowed autologous PRP preparation without causing any systemic problems. New Zealand rabbits are also easy to obtain and can be cared for at affordable prices. This makes them a favorable alternative in experimental studies investigating the biological effects of PRP. ${ }^{29}$

Finally, the platelet count method used in this study was important as well since there is no consensus regarding the ideal method to quantify this blood element. Studies have raised doubts about the level of agreement between manual and automated platelet counts. ${ }^{35-39}$ Although some authors have discussed the advantages of automated platelet counts, ${ }^{40}$ others have concluded that this method is less reliable than manual counts, especially in cases of thrombocytopenia. ${ }^{36,37}$ Therefore, manual platelet counts were used in the present study.

\section{CONCLUSIONS}

The controversies regarding the influence of PRP on wound healing should be re-examined, taking into consideration many variables including the protocol used for PRP preparation. Within the limits of this study, it can be concluded that the double-centrifugation protocol used in this study achieved higher platelet concentrations than did the single-centrifugation protocol used in this study. However, the double-centrifugation protocol caused alterations in platelet morphology and was more sensitive to small processing errors. Due to the small n numbers in each study group, the present results should be accepted as preliminary.

\section{REFERENCES}

1. Marx RE. Platelet-rich plasma: evidence to support its use. J Oral Maxillofac Surg 2004;62:489-496.

2. Anitua E. Plasma rich in growth factors: preliminary results of use in the preparation of sites for implants. Int $J$ Oral Maxillofac Implants 1999;14:529-535.
3. Man D, Plosker H, Winland-Brown JE. The use of autologous platelet-rich plasma (platelet gel) and autologous platelet-poor plasma (fibrin glue) in cosmetic surgery. Plast Reconstr Surg 2001;107:229-237.

4. Lekovic V, Camargo PM, Weinlaender M, Vasilic N, Kenney EB. Comparison of platelet-rich plasma, bovine porous bone mineral, and guided tissue regeneration versus platelet-rich plasma and bovine porous bone mineral in the treatment of intrabony defects: a reentry study. J Periodontol 2002;73:198-205.

5. Hanna R, Trejo PM, Weltman RL. Treatment of intrabony defects with bovine-derived xenograft alone and in combination with platelet-rich plasma: a randomized clinical trial. J Periodontol 2004;75:1668-1677.

6. Kassolis JD, Reynolds MA. Evaluation of the adjunctive benefits of platelet-rich plasma in subantral sinus augmentation. J Craniofac Surg 2005;16:280-287.

7. Aghaloo TL, Moy PK, Freymiller EG. Investigation of platelet-rich plasma in rabbit cranial defects: A pilot study. $J$ Oral Maxillofac Surg 2002;60:1176-1181.

8. Jakse N, Tangl S, Gilli R, Berghold A, Lorenzoni M, Eskici $A$, et al. Influence of PRP on autogenous sinus grafts. An experimental study on sheep. Clin Oral Implants Res 2003;14:578-583.

9. Choi BH, Im CJ, Huh JY, Suh JJ, Lee SH. Effect of platelet-rich plasma on bone regeneration in autogenous bone graft. Int J Oral Maxillofac Surg 2004;33:56-59.

10. Raghoebar GM, Schortinghuis J, Liem RS, Ruben JL, van der Wal JE, Vissink A. Does platelet-rich plasma promote remodeling of autologous bone grafts used for augmentation of the maxillary sinus floor? Clin Oral Implants Res 2005;16:349-356.

11. Anitua E, Sánchez M, Nurden AT, Nurden P, Orive G, Andía I. New insights into and novel applications for platelet-rich fibrin therapies. Trends Biotechnol 2006;24:227-234.

12. Weibrich G, Kleis WK, Hitzler WE, Hafner G. Comparison of the platelet concentrate collection system with the plasma rich in growth factors kit to produce platelet-rich plasma: a technical report. Int J Oral Maxillofac Implants 2005;20:118123.

13. Sánchez AR, Sheridan PJ, Kupp LI. Is platelet-rich plasma the perfect enhancement factor? A current review. Int $J$ Oral Maxillofac Implants 2003;18:93-103.

14. Whitman DH, Berry RL, Green DM. Platelet gel: an autologous alternative to fibrin glue with applications in oral and maxillofacial surgery. J Oral Maxillofac Surg 1997;55:12941299. 
15. Marx RE, Carlson ER, Eichstaedt RM, Schimmele SR, Strauss JE, Georgeff KR. Platelet-rich plasma: Growth factor enhancement for bone grafts. Oral Surg Oral Med Oral Pathol Oral Radiol 1998;85:638-646.

16. Tozum TF, Demiralp B. Platelet-rich plasma: a promising innovation in dentistry. J Can Dent Assoc 2003;69:664.

17. Weibrich G, Kleis WK, Hafner G, Hitzler WE, Wagner W. Comparison of platelet, leukocyte, and growth factor levels in point-of-care platelet-enriched plasma, prepared using a modified Curasan kit, with preparations received from a local blood bank. Clin Oral Implants Res 2003;14:357-362.

18. Landesberg R, Roy M, Glickman RS. Quantification of growth factor levels using a simplified method of platelet-rich plasma gel preparation. J Oral Maxillofac Surg 2000;58:297-300.

19. Sonnleitner D, Huemer P, Sullivan DY. A simplified technique for producing Platelet-rich plasma and Platelet Concentrate for intraoral bone grafting techniques: A technical note. Int J Oral Maxillofac Implants 2000;15:879-882.

20. Weibrich G, Kleis WK, Hafner G, Hitzler WE. Growth factor levels in platelet-rich plasma and correlations with donor age, sex, and platelet count. J Craniomaxillofac Surg 2002;30:97-102.

21. Tamimi FM, Montalvo S, Tresguerres I, Blanco Jerez L. A comparative study of 2 methods for obtaining platelet-rich plasma. J Oral Maxillofac Surg 2007;65:1084-1093.

22. Anitua E. La utilización de los factores de crecimiento plasmáticos en cirugía oral, maxilofacial y periodoncia (PRGF). Rev Actual Odontoestomatol 2001;6:305-315.

23. Eby EW. Platelet-rich plasma: Harvesting with a singlespin centrifuge. J Oral Implantol 2002;28:297-301.

24. Weibrich G, Kleis WK. Curasan PRP kit vs. PCCS PRP system. Collection efficiency and platelet counts of two different methods for the preparation of platelet-rich plasma. Clin Oral Implants Res 2002;13:437-443.

25. Weibrich G, Hansen T, Kleis W, Buch R, Hitzler WE. Effect of platelet concentration in platelet-rich plasma on periimplant bone regeneration. Bone 2004;34:665-671.

26. Grageda E. Platelet-rich plasma and bone graft materials: a review and a standardized research protocol. Implant Dent 2004;13:301-309.

27. Messora MR, Nagata MJH, Mariano RC, Dornelles RC, Bomfim SR, Fucini SE, et al. Bone healing in critical-size defects treated with platelet-rich plasma. A histologic and histometric study in rat calvaria. $J$ Periodontal Res 2008;43:217-223.

28. Kim SG, Kim WK, Park JC, Kim HJ. A comparative study of osseointegration of Avana implants in a demineralized freeze-dried bone alone or with platelet-rich plasma. $J$ Oral Maxillofac Surg 2002;60:1018-1025.
29. Efeoglu C, Akcay YD, Erturk S. A modified method for preparing platelet-rich plasma: an experimental study. J Oral Maxillofac Surg 2004;62:1403-1407.

30. Marx RE. Platelet-rich plasma (PRP): what is PRP and what is not PRP? Implant Dent 2001;10:225-228.

31. Dugrillon A, Eichler H, Kern S, Kluter H. Autologous concentrated platelet-rich plasma (cPRP) for local application in bone regeneration. Int J Oral Maxillofac Surg 2002;31:615619.

32. Schmitz G, Rothe G, Ruf A, Barlage S, Tschöpe D, Clemetson KJ, et al. European Working Group on Clinical Cell Analysis: Consensus protocol for the flow cytometric characterization of platelet function. Thromb Haemost 1998;79:885-896.

33. Michelson AD. Flow cytometry: A clinical test of platelet function. Blood 1996;87:4925-4936.

34. Halmay D, Sótonyi $P$, Vajdovich $P$, Gaál T. Morphological evaluation of canine platelets on Giemsa- and PAS-stained blood smears. Acta Vet Hung 2005;53:337-350.

35. Hatakeyama M, Beletti ME, Zanetta-Barbosa D, Dechich P. Radiographic and histomorphometric analysis of bone healing using autogenous graft associated with plateletrich plasma obtained by 2 different methods. Oral Surg Oral Med Oral Pathol Oral Radiol Endod 2008;105:e13-e18.

36. Kunz D. Possibilities and limitations of automated platelet counting procedures in the thrombocytopenic range. Semin Thromb Hemost 2001;27:229-235.

37. Langianni U, Limberti A, Bottari G, Ignesti C, Innocenti V. Evaluation of interferences in electronic platelet count. Quad Sclavo Diagn 1988;24:197-202.

38. Penev M, Kamenov V, Donkova O, Petkova D. Automatic and manual methods for counting the thrombocytes in the blood. Vutr Boles 1987;26:109-112.

39. Sutor AH, Grohmann A, Kaufmehl K, Wundisch, T. Problems with platelet counting in thrombocytopenia. A rapid manual method to measure low platelet counts. Semin Thromb Hemost 2001;27:237-243.

40. Woodell-May JE, Ridderman DN, Swift MJ, Higgins J. Producing accurate platelet counts for platelet rich plasma: validation of a hematology analyzer and preparation techniques for counting. J Craniofac Surg 2005;16:749-759. 